\title{
CASE REPORT: A RARE PRESENTATION OF DEEP VEIN THROMBOSIS WITH ACUTE HEMATOGENOUS OSTEOMYELITIS IN ADULT
}

Harsh Raval ${ }^{1}$, Neel Shah ${ }^{2}$, Jyotindra Pandit ${ }^{3}$, Prakash Amin ${ }^{4}$

\section{HOW TO CITE THIS ARTICLE:}

Harsh Raval, Neel Shah, Jyotindra Pandit, Prakash Amin. "A Rare Presentation of Deep Vein Thrombosis with Acute Hematogenous Osteomyelitis in Adult". Journal of Evolution of Medical and Dental Sciences 2015; Vol. 4, Issue 65, August 13; Page: 11423-11427, DOI: 10.14260/jemds/2015/1648

ABSTRACT: We report a case of 22 years old male presented with pain in left thigh, fever and dyspnea. On Imaging (MRI), an osteomyelitic focus in left thigh was noted. Venous Doppler had revealed a large thrombus in left lower limb. Curettage was done. After curettage patient become clinically asymptomatic. Staphyloccus aureus was identified from purulent material, Piperacilline and tazobactum antibiotics were given. It was a rare presentation of Deep Vein Thrombosis with Acute hematogenous Osteomyelitis in Adult. Cases are reported in Children.1-6 But not in Adults.

KEYWORDS: Deep Vein Thrombosis, Osteomyelitis, Adult, Staphylococcus Aureus.

INTRODUCTION: Deep vein thrombosis associated with Acute Hematogenous Osteomyelitis (Staphlococcus Aureus induced) is a rare entity in adults. Presentation of AHO with DVT is acute and severe as compared to cases without DVT. Studies mention that PVL (Panton-Valentine leukocidin) encoded strain of staphylococcus aureus is more often noted with DVT in case of AHO.7

CASE PRESENTATION: A 22 years male presented to clinic with pain left thigh since 15 days, difficulty in walking from last 10 days; fever, difficulty in breathing since last 3 days. Patient has denied any history of such symptoms and trauma before that. Then he developed pain over left thigh, which was dull aching, constant, mild to moderate in severity, increased with walking and routine activities, he was avoiding activities from last 15 days due to pain and remained in bed other than routine activities.

He was treated by oral and parenteral analgesics by family physician for pain. 10 days after starting of pain, he was examined and investigated. On Examination, local rise of temperature over the left thigh and tenderness noted. No tenderness over the left hip joint, no neurovascular deficits of left lower limb. Lab parameters were within acceptable limits, X-ray Left thigh (Figure 1) doesn't show any bony abnormalities, MRI (Figure 2, 3) of left thigh shows abnormal marrow signal density in upper midshaft of femur and cortical erosion of anteromedial aspect of upper shaft with intra cortical sclerotic cortical bone, adjacent soft tissue edema in muscular-intermuscular compartment with collection at medial aspect. He was started on parenteral cephalosporins under observation.

On $3^{\text {rd }}$ day of starting of treatment, patient was deteriorated with high grade fever, difficulty in breathing, and admitted to Intensive care unit. On admission, vital parameters temp- 101 degree $\mathrm{F}$, pulse 120/min, RR - 35/min, BP-100/60mmHg, Spo2- 85\%. He was put on PEEP mode on ventilator, Broad spectrum antibiotics and analgesics given. Laboratory noted a white blood cell count 11, 400 .

Platelet count of 550000, D-dimer 0.4, CRP-349mg/dl, PT-Test-18.1, INR-1.29 and APTTTest-38.9. Arterial Blood Gas Analysis pH-7.4, PCo2-33.3, Po2-71, Hco3-21.5. Chest X-ray (Figure 4)shows bilateral basal and mid zone soft tissues opacities.

Venous Doppler (Figure 5.1, 5.2) shows Deep vein thrombosis of left lower limb extending from left saphenous vein up to left iliac vein. 2D-Echo was not showing any sign of valve motion 
abnormalities. In spite of antibiotics and other measures, patient remained critical for 48 hours, so decision for curettage was taken. Before curettage, IVC filter was put. Under regional block, through anterolateral-Thompson's approach. ${ }^{8}$ between rectus femoris and vastus lateralis, anterolateral cortex of bone was approached.

Erosion of bone was noted, curettage was done with multiple drill holes, approximately $25 \mathrm{ml}$ of purulent material from medial aspect from soft tissues and $15 \mathrm{ml}$ of purulent material evacuated from medullary canal. Eroded cortex was removed. Purulent material and bone pieces were sent for culture sensitivity and histopathology respectively. Thorough wash was given with Normal saline. Gentamycin and Cefuroxime impregnated beads of PPMA was put inside the medullary cavity.

Within 12 hours of curettage, patient became clinically stable with vital parameters temp-98 degree F, pulse-86/min, RR-22/min, spo2- 97\% without oxygen. CRP came to $187 \mathrm{mg} / \mathrm{dl}$ and on subsequent day it became $90 \mathrm{mg} / \mathrm{dl}$.

After 12 hours of surgery, patient was started on Fondaperinaux-anti coagulant; platelet count came to 120000 by 24 hours of starting anticoagulants.

Organism Staphylococcus Aureus was isolated and started on Piperacillin and Tazobactum as it was most sensitive.

Patient was discharged by advising parenteral antibiotics for 3 weeks, Oral anticoagulants.

On follow up after 6 weeks patient is much better now. Surgical wound has healed well and patient is not having any pain or difficulty walking.

DISCUSSION: Venous thrombosis is rare in adults 2 to $7 / 10,000$ patients. The locations of the infections in the patients with deep venous thrombosis exclusively involved the spine, pelvis, and lower extremities, and most of the infections occurred adjacent to the major vascular structures of the lower extremities. ${ }^{2}$

One conclusive study by Hollmig. ${ }^{2}$ suggest that in patient of Acute hematogenous osteomyelitis with Venous Thrombosis, presentation of disease is more acute and severe as compared to patients who has having AHO without DVT.

S. aureus septicemia presents a rare but potentially serious cause of DVT. Septicemia triggers thrombosis through the processes described in Virchow's triad: Stasis, Hypercoagulability and Endothelial Damage. More specifically, exotoxins released by S. aureus cause the aggregation of platelets and spasm of smooth muscles through their association with cell membranes. Coagulation is supported further by the release of local staphylococcal enzymes (like coagulase) which cause clot formation by interacting with fibrinogen. Through these processes, a localized infection, like osteomyelitis, creates an environment that is favorable to the formation of an adjoining DVT.9,10

There is some evidence suggesting that the presence of the PVL gene encoded in strains of methicillin-resistant Staphylococcus aureus and methicillin-sensitive Staphylococcus aureus may explain the occurrence of complications such as deep Venous thrombosis associated with deep musculoskeletal infection. Martinez-Aguilar et al. recently reported that the Panton-Valentine leukocidin (PVL) gene was encoded in the strains of Staphylococcus aureus isolated from all five children who had development of deep venous thrombosis in a series of twenty-eight children with musculoskeletal infection. ${ }^{3}$

As noted, DVT associated with S. aureus septicemia is rare, Existent case reports are uncommon, but demonstrate serious complication rates of up to $33 \%$. 


\section{CASE REPORT}

CONCLUSION: In conclusion, the risk of DVT is always to be borne in mind in case of AHO, especially when the presentation is acute and severe, with very strong local and significant general signs. In presence of pulmonary lesions suggestive of septic emboli, exploration for DVT is also mandatory. If it is discovered, treatment should be energetic and surveillance tight; low molecular-weight heparin should be administered to prevent local extension of thrombosis and embolism. Use of intravascular filters is effective.

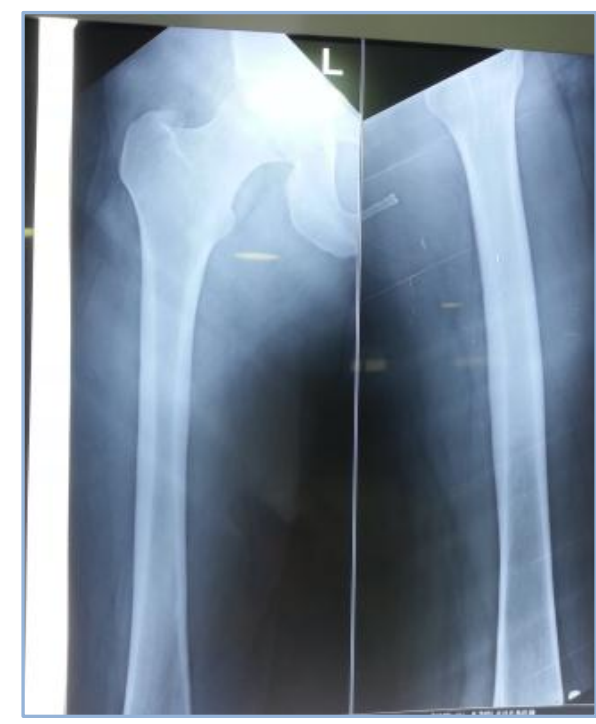

Fig. 1: X-ray (L) Femur AP/Lat

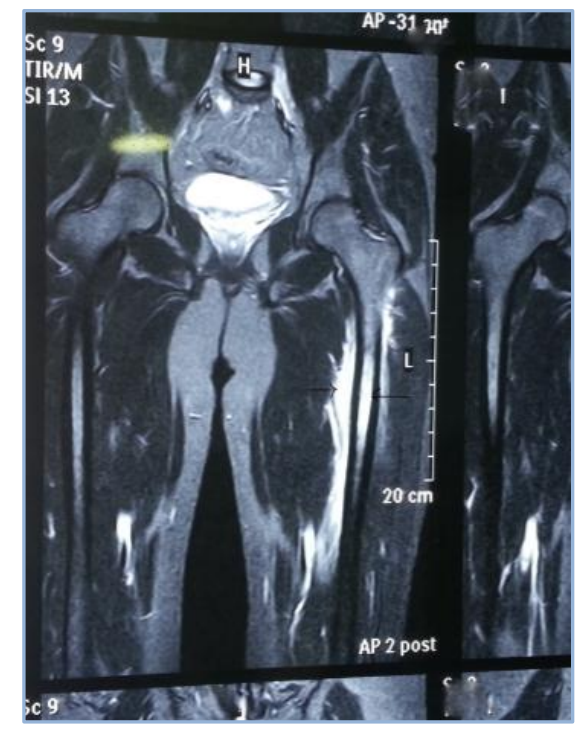

Fig. 2: MRI Antero medial Aspect of Femur showing High Signal Density

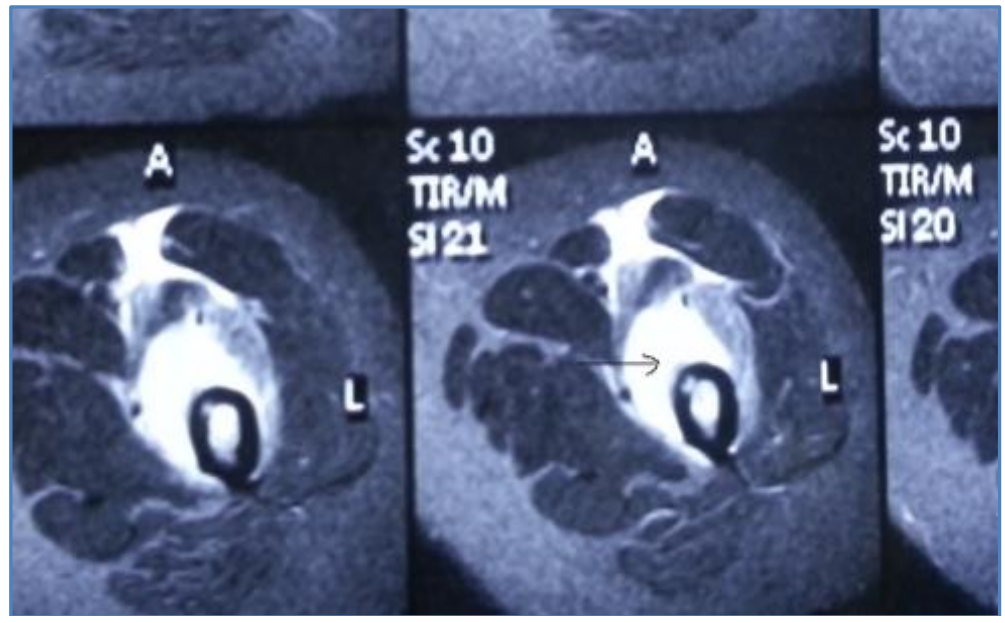

Fig. 3: MRI Showing Cortical Sclerotic lesion 


\section{CASE REPORT}

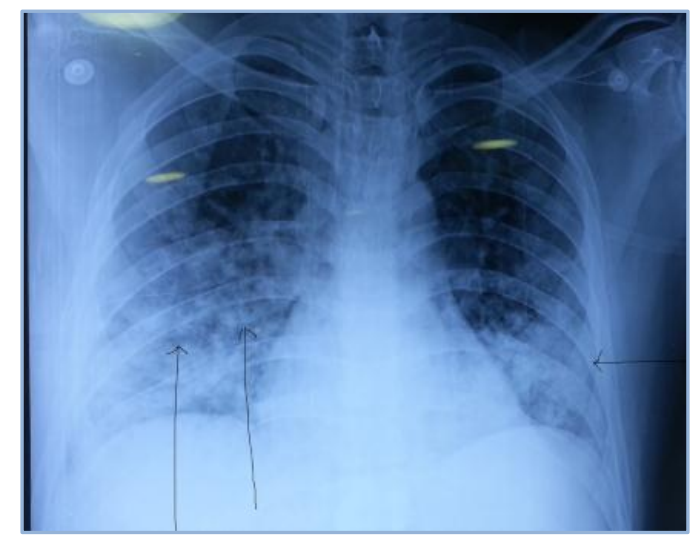

Fig. 4: Shows Bilateral Basal and mid zone Soft Tissues Opacities

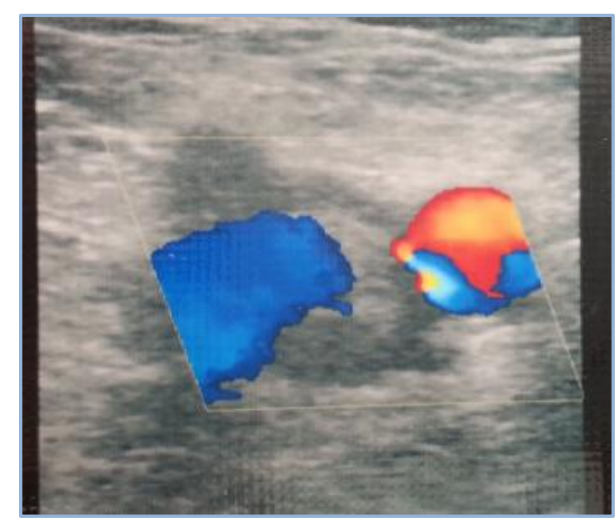

Fig. 5.1

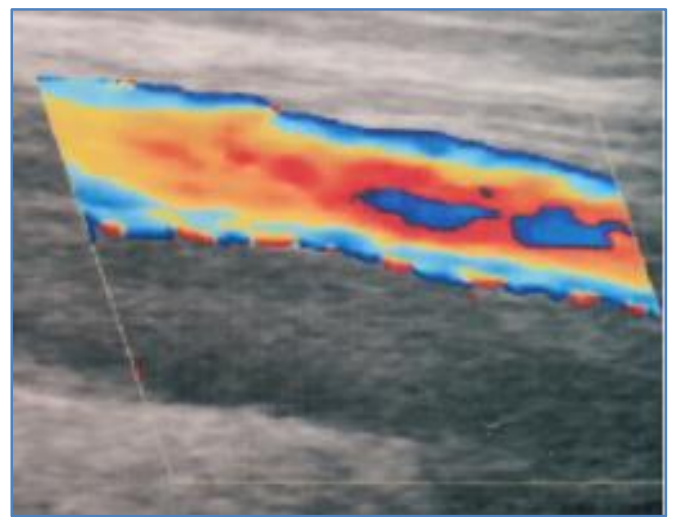

Fig. 5.2

\section{REFERENCES:}

1. Oberdorfer $\mathrm{P}$, Odell S, Kongthavonsakul K. Deep vein thrombosis associated with Staphylococcus aureus septicemia. Chiang Mai Medical Journal 2012; 51(3): 87-91.

2. Hollmig S.T., Copley L.A., Browne R.H., Grande L.M., Wilson P.L. Deep venous thrombosis associated with osteomyelitis in children. J Bone Joint SurgAm 2007; 89: 1517-1523. Journal article.

3. Gite A., Trivedi R., Ali U.S. Deep vein thrombosis associated with osteomyelitis. Indian Pediatr. 2008 May; 45(5): 418-419.

4. Bouchoucha S., Benghachame F., Trifa M. Deep venous thrombosis associated with acute hematogenous osteomyelitis in children. Orthop Traumatol Surg Res. 2010 Dec; 96(8): 890893.

5. Gonzalez B.E., Teruya Jun, Mahoney D.H., Jr. Venous thrombosis associated with Staphylococcal osteomyelitis in children. Pediatrics. 2006; 117: 167. 


\section{CASE REPORT}

6. Yuksel H., Ozguven A.A., Akil I., Erguder I., Yilmaz D., Cabuk M. Septic pulmonary emboli presenting with deep venous thrombosis secondary to acute osteomyelitis. Pediatr Int. 2004; 46: 621-623.

7. Martinez-Aguilar G, Avalos-Mishaan A, Hulten K, Hammerman W, Mason EO Jr, Kaplan SL. Community-acquired, methicillin-resistant and methicillin-susceptible Staphylococcus aureus musculoskeletal infections in children. Pediatr Infect Dis J. 2004; 23: 701-6. Journal article.

8. Campbell's Operative Orthopaedics, 11th ed; Part 1- General principles, Surgical Approaches, Page 53.

9. Prasad R, Mishra O.P,Pant P. Deep vein thrombosis with Staphylococcus aureus septicemia. Indian Pediatr. 2007 Jan; 44(1): 43-45.

10. Gorenstein A, Gross E, Houri S, Gewirts G, Katz S. The pivotal role of deep vein thrombophlebitis in the development of acute disseminated Staphylococcal disease in children. Pediatrics. 2000; 106(6).

\section{AUTHORS:}

1. Harsh Raval

2. Neel Shah

3. Jyotindra Pandit

4. Prakash Amin

\section{PARTICULARS OF CONTRIBUTORS:}

1. Junior Consultant, Department of Orthopaedics, Harikrupa Orthopaedic Hospital.

2. Resident, Department of Orthopaedics, Inlaks and Budhrani Hospital.

3. Senior Consultant, Department of Orthopaedics, Harikrupa Orthopaedic Hospital.

FINANCIAL OR OTHER COMPETING INTERESTS: None
4. Senior Consultant, Department of Orthopaedics, Harikrupa Orthopaedic Hospital.

\section{NAME ADDRESS EMAIL ID OF THE CORRESPONDING AUTHOR:}

Dr. Neel Shah, Resident, Department of Orthopedics, Inlaks and Badhurani-411001, Maharashtra.

E-mail: shahneel89@yahoo.com

Date of Submission: 19/07/2015.

Date of Peer Review: 20/07/2015.

Date of Acceptance: 07/08/2015.

Date of Publishing: 13/08/2015. 